\title{
MOTIONS IN LINEARLY CONNECTED TWO-DIMENSIONAL SPACES
}

JACK LEVINE

1. Introduction. A linearly connected space $L_{n}$ is characterized by its coefficients of (asymmetric) connection $L_{j \mathrm{k}}^{\mathfrak{j}}$ which transform according to the law $[2, \text { p. 3 }]^{1}$

$$
L_{j k}^{i}(x) \frac{\partial \bar{x}^{a}}{\partial x^{i}}=\bar{L}_{b c}^{a}(\bar{x}) \frac{\partial \bar{x}^{b}}{\partial x^{j}} \frac{\partial \bar{x}^{c}}{\partial x^{k}}+\frac{\partial^{2} \bar{x}^{a}}{\partial x^{j} \partial x^{k}} .
$$

An $L_{n}$ will admit an infinitesimal motion defined by the infinitesimal transformation

$$
\bar{x}^{i}=x^{i}+\xi^{i}(x) \delta t
$$

if

(1.3a) $\frac{\partial^{2} \xi^{i}}{\partial x^{j} \partial x^{k}}+\frac{\partial \Gamma_{j k}^{i}}{\partial x^{h}} \xi^{h}+\Gamma_{h k}^{i} \frac{\partial \xi^{h}}{\partial x^{j}}+\Gamma_{j h}^{i} \frac{\partial \xi^{h}}{\partial x^{k}}-\Gamma_{j k}^{h} \frac{\partial \xi^{i}}{\partial x^{h}}=0$,

$$
\frac{\partial \Omega_{j k}^{i}}{\partial x^{h}} \xi^{h}+\Omega_{h k}^{i} \frac{\partial \xi^{h}}{\partial x^{j}}+\Omega_{j h}^{i} \frac{\partial \xi^{h}}{\partial x^{k}}-\Omega_{j k}^{h} \frac{\partial \xi^{i}}{\partial x^{h}}=0,
$$

where

$$
L_{j k}^{i}=\Gamma_{j k}^{i}+\Omega_{j k}^{i},
$$

and $\Gamma_{j k}^{j}, \Omega_{j k}^{i}$ are the symmetric and skew-symmetric parts respectively of $L_{j k}^{i}[1$, p. 231].

In case $\Omega_{j k}^{i}=0$, the $L_{n}$ reduces to a symmetrically connected space $A_{n}$ in which (1.3a) defines the infinitesimal affine collineations [2, p. 125], so that motions in an $L_{n}$ can be considered as generalizations of such collineations of an $A_{n}$ [3].

In a previous paper [3] we obtained all (symmetric) two-dimensional spaces $A_{2}$ admitting real continuous groups of affine collineations, these being obtained by solving (1.3a) for the $\Gamma_{j k}^{1}$, the $\xi^{i}$ being known and obtained from Lie's classification [4, pp. 71-73, 379-380] giving all real continuous groups $G_{r}$ in two variables. In $\$ 2$ we carry through a like procedure to obtain all linearly connected $L_{2}$ admitting complete groups of motions. This will involve the solution of the

Received by the editors November 27, 1950.

${ }^{1}$ Numbers in brackets refer to the bibliography at the end of the paper. 
combined system $(1.3 \mathrm{a}-\mathrm{b})$ for the $\Gamma_{j k}^{i}$ and $\Omega_{j k}^{i}$. The results of [3] will of course eliminate much of the analysis which would otherwise be necessary. We shall exclude all solutions of the system (1.3) which lead to $A_{2}$ spaces $\left(\Omega_{j k}^{i}=0\right)$.

In $\$ 3$ we consider the special case of spaces of absolute parallelism $T_{n}$, and determine all $T_{2}$ admitting motion groups. The conditions on an $L_{n}$ to reduce to a $T_{n}$ are $[1$, p. 234]

$$
L_{j k m}^{i} \equiv \frac{\partial L_{j m}^{i}}{\partial x^{k}}-\frac{\partial L_{j k}^{i}}{\partial x^{m}}+L_{j m}^{h} L_{h k}^{i}-L_{j k}^{h} L_{h m}^{i}=0,
$$

that is, the $L_{n}$ is of zero curvature. In this case there exists an ennuple of (absolutely) parallel vector fields $h_{\alpha \mid}^{i}$, that is,

$$
h_{\alpha \mid j}^{i} \equiv \frac{\partial h_{\alpha \mid}^{i}}{\partial x^{j}}+h_{\alpha \mid}^{m} L_{m j}^{i}=0 \quad(\alpha=1, \cdots, n) .
$$

These equations (1.6) are solved for the $h_{\alpha \mid}^{4}$ using for the $L_{j \mathbf{k}}^{i}$ the values found from the solutions of (1.3). (The integrability conditions of (1.6) are (1.5).)

The problem of motions in a $T_{n}$ has been treated by Robertson [5] who obtained the conditions for infinitesimal motions of a $T_{n}$ in the form

$$
\frac{\partial h_{\alpha \mid}^{i}}{\partial x^{j}} \xi^{j}-h_{\alpha \mid}^{j} \frac{\partial \xi^{i}}{\partial x^{j}}=\underset{\epsilon_{\alpha}}{\beta} h_{\beta \mid}^{i}
$$

where $\epsilon_{\alpha}^{\beta}$ are constants. (See also [6].)

It is shown in $\$ 2$ that there are 8 possible types of $L_{2}$ admitting groups of motions, the maximum number of parameters being four. In $\$ 3$ it is shown there are 7 types of motions for a $T_{2}$.

2. Motions in an $L_{2}$. We proceed to the solution of (1.3) for the case $n=2$. It is easily shown that $(1.3 \mathrm{~b})$ reduces to

$$
\begin{aligned}
& \frac{\partial A}{\partial x} \xi^{1}+\frac{\partial A}{\partial y} \xi^{2}+A \frac{\partial \xi^{2}}{\partial y}-B \frac{\partial \xi^{1}}{\partial y}=0 \\
& \frac{\partial B}{\partial x} \xi^{1}+\frac{\partial B}{\partial y} \xi^{2}+B \frac{\partial \xi^{1}}{\partial x}-A \frac{\partial \xi^{2}}{\partial x}=0
\end{aligned}
$$

where

$$
A=\Omega_{12}^{1}, \quad B=\Omega_{12}^{2} \quad\left(x, y=x^{1}, x^{2}\right) .
$$


Corresponding to each $G_{r}$ of Lie's classification we solve (2.1) for $A$ and $B$, excluding all solutions for which $A=B=0$. (The solutions of (1.3a) for the $\Gamma_{j k}^{i}$ have been obtained in [3].) For each of the nonexcluded solutions the $G_{r}$ is tested for completeness as explained in [3].

As an illustration we consider the $G_{3}[p, q, x q]$ where $p=\partial f / \partial x$, $q=\partial f / \partial y$. Using $X_{1} f=p, X_{2} f=q, X_{3} f=x q$, with $\xi_{1 \mid}^{i}=\delta_{1}^{i}, \xi_{2 \mid}^{i}=\delta_{2}^{i}, \xi_{3 \mid}^{1}=0$, $\xi_{3 \mid}^{2}=x$ in (2.1) we obtain $A=0, B=B_{0}$ an arbitrary constant.

To obtain the complete group, put $A=0, B=B_{0}$ in (2.1), and solve for $\xi^{i}$. This gives $\xi^{1}=k, \xi^{2}=\xi^{2}(x, y)$, with $k$ an arbitrary constant and $\xi^{2}$ an arbitrary function. Now as determined in [3] the $\Gamma$ 's obtained as solutions of (1.3a) corresponding to the above $G_{3}$ will admit as their complete group the $G_{4}[p, q, x q, y q]$. It is thus seen that by taking $k=0, \xi^{2}=y$, we obtain the fourth generator $y q$ of the $G_{4}$, and hence this $G_{4}$ will be the complete group of motions of the $L_{2}$ given by

$$
\begin{array}{ll}
L_{11}^{1}=2 a, & L_{12}^{1}=L_{21}^{1}=L_{22}^{1}=L_{11}^{2}=L_{22}^{2}=0, \\
L_{12}^{2}=a+B_{0}, & L_{21}^{2}=a-B_{0} \quad\left(B_{0} \neq 0\right) .
\end{array}
$$

The $\Gamma$ 's used to give these $L$ 's are obtained from group [4.1] of [3].

By proceeding as above with each $G_{r}$ and making use of the results of [3] we find eight possible types of $L_{2}$ spaces admitting real groups of motions. These with their respective groups are given below.

Asymmetrically connected space $L_{2}$ and their complete groups of motions [A1.1] [p]

[A2.1] $[p, q]$

$$
L_{j k}^{i}=L_{j k}^{i}(y)
$$

(arbitrary functions of $y$ ).

$$
L_{j k}^{i}=\text { arbitrary constants. }
$$

[A2.2] $[p, x p+y q]$

$$
L_{j k}^{i}=(1 / y) A_{j k}^{i} \quad\left(A_{j k}^{i} \text { arbitrary constants }\right) .
$$

[A3.1] $\left[p, 2 x p+y q, x^{2} p+x y q\right]$

$$
\begin{aligned}
& L_{11}^{1}=\frac{2 b}{y^{2}}, \quad L_{12}^{1}=-\frac{1}{y}, \quad L_{21}^{1}=-\frac{1}{y}, \quad L_{22}^{1}=0, \\
& L_{11}^{2}=\frac{a}{y^{3}}, L_{12}^{2}=\frac{b+c}{y^{2}}, L_{21}^{2}=\frac{b-c}{y^{2}}, L_{22}^{2}=-\frac{2}{y} \quad(c \neq 0) .
\end{aligned}
$$


[A3.2] $[q, x q, y q]$

$$
\begin{aligned}
& L_{11}^{1}=2 f(x), \quad L_{12}^{1}=L_{21}^{1}=L_{22}^{1}=L_{11}^{2}=L_{22}^{2}=0, \\
& L_{12}^{2}=f(x)+B(x), \quad L_{21}^{2}=f(x)-B(x) \quad(B \neq 0) .
\end{aligned}
$$

[A4.1] $[p, q, x q, y q]$

$$
\begin{aligned}
& L_{11}^{1}=2 a, \quad L_{12}^{1}=L_{21}^{1}=L_{22}^{1}=L_{11}^{2}=L_{22}^{2}=0, \\
& L_{12}^{2}=a+b, \quad L_{21}^{2}=a-b \\
& (b \neq 0) \text {. }
\end{aligned}
$$

[A4.2] $\left[p, q, y q, e^{x} q\right]$

$$
\begin{aligned}
& L_{11}^{1}=1 / 3+2 a, \quad L_{12}^{1}=L_{21}^{1}=L_{22}^{1}=L_{11}^{2}=L_{22}^{2}=0, \\
& L_{12}^{2}=a+c-1 / 3, \quad L_{21}^{2}=a-c-1 / 3 \quad(c \neq 0) .
\end{aligned}
$$

[A4.3] [p,yq, $\left.\left(e^{a x} \cos x\right) q, \quad\left(e^{a x} \sin x\right) q\right]$

$$
\begin{aligned}
& L_{11}^{1}=2 a / 3+2 b, \quad L_{12}^{1}=L_{21}^{1}=L_{22}^{1}=L_{22}^{2}=0 \text {, } \\
& L_{11}^{2}=\left(1+a^{2}\right) y, \quad L_{12}^{2}=-2 a / 3+b+c \text {, } \\
& L_{21}^{2}=-2 a / 3+b-c \\
& (c \neq 0) .
\end{aligned}
$$

In the above $a, b, c$ are arbitrary constants, $f(x), B(x)$ are arbitrary functions subject to indicated restrictions.

3. Motions in spaces $T_{2}$ of absolute parallelism. For each of the 8 types of $L_{2}$ obtained in the previous section, we impose conditions (1.5), and then solve (1.6) for the $h_{\alpha \mid}^{i}$.

The case [A3.2] will be used as an illustration. Conditions (1.5) reduce to $(f+B)^{\prime}-(f+B)^{2}=0$, so that $f(x)+B(x)=-1 /(x+c)$ with $c$ an arbitrary constant. Equations (1.6) are
(3.1a) $\frac{\partial h^{1}}{\partial x}+2 h^{1} f(x)=0$,
(3.1c) $\frac{\partial h^{2}}{\partial x}+h^{2}(f(x)-B(x))=0$,

$$
\frac{\partial h^{1}}{\partial y}=0,
$$

$$
\frac{\partial h^{2}}{\partial y}-\frac{h^{1}}{x+c}=0,
$$

which can be solved to give $h^{1}=a g(x), h^{2}=(a y+b) g(x) /(x+c)$, where $g(x)=\exp \left\{-2 \int f(x) d x\right\}$.

The complete solution is then

$$
h_{\alpha \mid}^{1}=a_{\alpha} g(x), \quad h_{\alpha \mid}^{2}=\frac{a_{\alpha} y+b_{\alpha}}{x+c} g(x) \quad(\alpha=1,2),
$$

with $a_{\alpha}, b_{\alpha}, c$ arbitrary constants such that $a_{1} b_{2}-a_{2} b_{1} \neq 0$. (The special case $L_{12}^{2}=f+B=0$ gives the solution $h_{\alpha \mid}^{1}=a_{\alpha} g(x), h_{\alpha \mid}^{2}=b_{\alpha} g(x)$.) 
It is of interest to verify that the $T_{2}$ will admit the finite group generated by the infinitesimal group. To show this we first obtain the finite equations of the group, these being

$$
\bar{x}=x, \quad \bar{y}=\lambda x+\mu y+\nu
$$

with the 3 parameters $\lambda, \mu, \nu$.

Now we must have [5]

$$
h_{\alpha \mid}^{i}(\bar{x}, \bar{y})=A_{\alpha}^{\beta} h_{\beta \mid}^{j}(x, y) \frac{\partial \bar{x}^{i}}{\partial x^{j}},
$$

where the $A_{\alpha}^{\beta}$ must be functions $A_{\alpha}^{\beta}(\lambda, \mu, \nu)$ of the parameters only. From (3.2), (3.3), and (3.4) we obtain the relations

$$
A_{\alpha}^{\beta} a_{\beta}=a_{\alpha}, \quad A_{\alpha}^{\beta} b_{\beta}=c_{\alpha},
$$

where $c_{\alpha}=\left[a_{\alpha}(\nu-\lambda c)+b_{\alpha}\right] / \mu$. It follows that the $A_{\alpha}^{\beta}$ exist and are of the required form.

The results from the solutions of (1.6) are given below.

Spaces $T_{2}$ of absolute parallelism admitting complete groups of motions [T1.1] [p]

(a) $h_{\alpha \mid}^{1}=a_{\alpha}^{j} \exp \left\{\int\left(\lambda_{j} L_{2 i}^{1}-L_{1 i}^{1}\right) d x^{i}\right\}$,

$$
h_{\alpha \mid}^{2}=-\sum_{j} a_{\alpha}^{j} \lambda_{j} \exp \left\{\int\left(\lambda_{j} L_{2 i}^{1}-L_{1 i}^{1}\right) d x^{i}\right\}
$$

(b) $h_{\alpha \mid}^{1}=\exp \left\{\int\left(\lambda L_{2 i}^{1}-L_{1 i}^{1}\right) d x^{i}\right\}\left[a_{\alpha} \cos \rho+b_{\alpha} \sin \rho\right]$,

$$
\begin{array}{r}
h_{\alpha \mid}^{2}=-\exp \left\{\int\left(\lambda L_{2 i}^{1}-L_{1 i}^{1}\right) d x^{i}\right\}\left[a_{\alpha}(\lambda \cos \rho-\mu \sin \rho)\right. \\
\left.+b_{\alpha}(\lambda \sin \rho+\mu \cos \rho)\right]
\end{array}
$$

(c) $h_{\alpha \mid}^{1}=\exp \left\{\int\left(\lambda L_{2 i}^{1}-L_{1 i}^{1}\right) d x^{i}\right\}\left[a_{\alpha} \int \frac{L_{2 i}^{1} d x^{i}}{L_{21}^{1}}+b_{\alpha}\right]$,

$$
\begin{aligned}
& h_{\alpha \mid}^{2}=-\exp \left\{\int\left(\lambda L_{2 i}^{1}-L_{1 i}^{1}\right) d x^{i}\right\} \\
& \cdot\left[\frac{a_{\alpha}}{L_{21}^{1}}+\lambda\left(a_{\alpha} \int \frac{L_{2 i}^{1} d x^{i}}{L_{21}^{1}}+b_{\alpha}\right)\right] .
\end{aligned}
$$

In (a), $\lambda_{1} \neq \lambda_{2}$ are the real roots of $f(\lambda) \equiv \lambda^{2} L_{21}^{1}+\lambda\left(L_{21}^{2}-L_{11}^{1}\right)-L_{11}^{2}=0$; 
in (b), $\lambda \pm i \mu$ are its roots; and in (c), $f(\lambda)$ is assumed to have equal roots, $\lambda, \lambda$. In (b), $\rho=\int \mu L_{2 i}^{1} d x^{i}$. The $L_{j k}^{i}(y)$ are otherwise arbitrary subject to (1.5), which have as a consequence that $L_{11}+L_{21}$ and $L_{11} L_{21}-L_{21} L_{11}$ must be constant. The $a_{\alpha}, b_{\alpha}, a_{\alpha}^{j}$ are arbitrary constants such that $h_{\alpha \mid}^{i}$ are linearly independent. For special values of the $L_{j k}^{i}$, for example, $L_{21}^{1}=0$, the solutions for the $h_{\alpha \mid}^{i}$ can be easily obtained.

[T2.1] $[p, q]$

The $h_{\alpha \mid}^{4}$ are obtained as in the 3 cases of [T1.1] except the $L_{j k}^{\mathbf{k}}$ are now considered constants.

[T2.2] $[p, x p+y q]$

$h_{\alpha \mid}^{1}=y^{m}\left(a_{\alpha} u+b_{\alpha}\right), \quad h_{\alpha \mid}^{2}=y^{m}\left(c_{\alpha} v+d_{\alpha}\right)$,

with $c_{\alpha} A_{11}^{2}+a_{\alpha} A_{11}^{1}=0, d_{\alpha} A_{21}^{1}+b_{\alpha} A_{11}^{1}=0, m=-\left(1+A_{12}^{1}+A_{22}^{2}\right) / 2$, and $u=x A_{21}^{1}+y A_{22}^{1}, v=x A_{11}^{2}+y A_{12}^{2}$. The constants $A_{j k}^{t}$ are arbitrary subject to (1.5). As in [T1.1] the solutions for $h_{\alpha \mid}^{i}$ corresponding to special values of the $A_{j k}^{j}$ can be obtained without difficulty.

[T3.1] $\left[p, 2 x p+y q, x^{2} p+x y q\right]$

$$
h_{\alpha \mid}^{1}=y\left(a_{\alpha} x+b_{\alpha}\right), \quad h_{\alpha \mid}^{2}=\left(y^{2}+2 b x\right) a_{\alpha}+2 b b_{\alpha} .
$$

The $a, b, c$ of [A3.1] must satisfy $c=3 b, a=4 b^{2}, b \neq 0$.

[T3.2] $[q, x q, y q]$

$$
h_{\alpha \mid}^{1}=a_{\alpha} g(x), \quad h_{\alpha \mid}^{2}=\left(a_{\alpha} y+b_{\alpha}\right) g(x) /(x+c) .
$$

Here the $L_{j k}^{4}$ of [A3.2] satisfy $g(x)=\exp \left\{-\int 2 f(x) d x\right\}$, and $f(x)$ $+B(x)=-1 /(x+c)$.

[T4.1] $[p, q, x q, y q]$

$$
h_{\alpha \mid}^{1}=a_{\alpha} e^{-2 a x}, \quad h_{\alpha \mid}^{2}=b_{\alpha} e^{-2 a x},
$$

with $a+b=0,(b \neq 0)$ in [A4.1].

[T4.2] $\left[p, q, y q, e^{x} q\right]$

$$
\begin{aligned}
& h_{\alpha \mid}^{1}=a_{\alpha} e^{(2 c-1) x}, h_{\alpha \mid}^{2}=b_{\alpha} e^{2 c x} \quad(a+c=1 / 3 \text { in [A4.2]), } \\
& h_{\alpha \mid}^{1}=a_{\alpha} e^{(2 c+1) x}, h_{\alpha \mid}^{2}=\left(a_{\alpha} y+b_{\alpha}\right) e^{(2 c+1) x} \\
&(a+c=-2 / 3 \text { in [A4 2] }) .
\end{aligned}
$$

\section{BIBLIOGRAPHY}

1. L. P. Eisenhart, Continuous groups of transformations, Princeton University Press, 1933. 
2. - Non-Riemannian geometry, Amer. Math. Soc. Colloquium Publications, vol. 8, 1927.

3. J. Levine, Classification of collineations in projectively and affinely connected spaces of troo dimensions, Ann. of Math. vol. 52 (1950) pp. 465-477.

4. S. Lie and F. Engel, Theorie der Transformationsgruppen, vol. 3, Leipzig, Teubner, 1893.

5. H. P. Robertson, Groups of motions in spaces admitting absolute parallelism, Ann. of Math. vol. 33 (1932) pp. 496-520.

6. K. Yano, Groups of transformations in generalized spaces, Tokyo, Akademeia Press, 1949.

North Carolina State College 